\title{
Selection by current compliance of negative and positive bipolar resistive switching behaviour in $\mathrm{ZrO}_{2-\mathrm{x}} / \mathrm{ZrO}_{2}$ bilayer memory
}

\author{
Ruomeng Huang, Xingzhao Yan, Katrina A Morgan, Martin D B Charlton, and C H \\ (Kees) de Groot \\ Nanoelectronics and Nanotechnology Group, Department of Electronics and Computer Science, \\ University of Southampton, SO17 1BJ, UK \\ E-mail: R.Huang@ soton.ac.uk
}

\begin{abstract}
We report here a $\mathrm{ZrO}_{2-\mathrm{x}} / \mathrm{ZrO}_{2}$-based bilayer resistive switching memory with unique properties that enables the selection of the switching mode by applying different electroforming current compliances. Two opposite polarity modes, positive bipolar and negative bipolar, correspond to the switching in the $\mathrm{ZrO}_{2}$ and $\mathrm{ZrO}_{2-x}$ layer, respectively. The $\mathrm{ZrO}_{2}$ layer is proved to be responsible for the negative bipolar mode which is also observed in $\mathrm{ZrO}_{2}$ single layer device. The oxygen deficient $\mathrm{ZrO}_{2-\mathrm{x}}$ layer plays the dominant role in the positive bipolar mode, which is exclusive to the bilayer memory. A systematic investigation of the $\mathrm{ZrO}_{2-\mathrm{x}}$ composition in the bilayer memory suggests that $\mathrm{ZrO}_{1.8}$ layer demonstrates optimum switching performance with low switching voltage, narrow switching voltage distribution and good cycling endurance. An excess of oxygen vacancies, beyond this composition, leads to a deterioration of switching properties. The formation and dissolution of the oxygen vacancy filament model has been proposed to explain both polarity switching behaviours and the improved properties in the bilayer positive bipolar mode are attributed to the confined oxygen vacancy filament size within the $\mathrm{ZrO}_{2-x}$ layer.
\end{abstract}

Keywords: resistive random access memory, bilayer, $\mathrm{ZrO}_{2-\mathrm{x}}$, oxygen vacancy

\section{Introduction}

Transition metal oxides based resistive random access memory (RRAM) has attracted great attention as the leading candidate to replace flash memory due to their advantages of simple structure, remarkable scalability, and excellent memory performances [1]. A variety of metal oxides, including $\mathrm{TiO}_{2}, \mathrm{HfO}_{2}, \mathrm{ZnO}$, have been extensively investigated as the switching layer in RRAM [2-9]. Recently, $\mathrm{ZrO}_{2}$-based RRAM has become increasingly investigated because of its high thermodynamic stability, large bandgap, high dielectric constant and compatibility with the CMOS process [10-13]. Both unipolar and bipolar switching behaviour based on $\mathrm{ZrO}_{2}$ have been reported in previous works [14-17].

Despite its promising future, great challenges including the wide resistance distribution as well as the non-uniformity of the SET and RESET voltages still remain to be tackled before RRAM can be industrialised. Bilayer structure RRAM devices have been proposed to improve the switching performance by reducing the switching power and improving the switching uniformity. Several bilayer combinations such as $\mathrm{TiO}_{2-x} / \mathrm{TiO}_{2-y}[18,19], \mathrm{Ta}_{2} \mathrm{O}_{5-x} / \mathrm{TaO}_{2-x}[20]$, $\mathrm{ZrO}_{\mathrm{x}} / \mathrm{HfO}_{\mathrm{y}}[21]$ and $\mathrm{HfO}_{2} / \mathrm{TiO}_{2}$ [22] have been realised with enhanced switching properties. Although the detailed switching mechanism of RRAM remains uncertain, it is widely accepted that the migration of oxygen vacancies under an applied electrical field are responsible for the switching behaviour [23]. The improved performance in the bilayer structure is thought to be 
associated with the confined/localized oxygen vacancy filament at the bilayer interface [21,22]. Recent studies on single oxide layer based RRAM have suggested that the oxygen concentration within the transition metal oxide plays a significant role in the memory switching properties [24,25]. However, systematic analysis of the oxide concentration effect on the bilayer structural RRAM is still lacking.

In this study, we demonstrated the first $\mathrm{ZrO}_{2-\mathrm{x}}$-based bilayer RRAM devices. By applying different electroforming current compliance, the switching location within the bilayer $\mathrm{TiN} / \mathrm{ZrO}_{2-\mathrm{x}} / \mathrm{ZrO}_{2} / \mathrm{TiN}$ memory device can be programmed into either the $\mathrm{ZrO}_{2}$ or the $\mathrm{ZrO}_{2-\mathrm{x}}$ layer, both of which exhibit opposite switching polarities. An improved switching performance was observed in the $\mathrm{ZrO}_{2-\mathrm{x}}$ layer related positive bipolar mode, which was proved to be strongly dependent on the oxygen concentration of the $\mathrm{ZrO}_{2-x}$ layer.

\section{Experiment details}

Zirconium oxide thin films were prepared by medium frequency plasma assisted magnetron sputtering (Leybold Optics HELIOS Pro XL) at room temperature. In this process, the substrate was rotating at a speed of $180 \mathrm{rpm}$ to ensure a uniform deposition. During each rotation, a thin layer of $\mathrm{Zr}$ was firstly deposited from a $\mathrm{Zr}$ metal targets (99.99\% purity) using a power of $2000 \mathrm{~W}$ in an Ar atmosphere. This thin film was transformed into an oxide layer by passing the substrate underneath the $\mathrm{O}_{2}$ plasma of the RF source. Different $\mathrm{O}_{2}$ flow rates $(6 \mathrm{sccm}$ to 20 $\mathrm{sccm}$ ) were used in this work to obtain Zirconium oxide films with different stoichiometry.

The deposited films were investigated using scanning electron microscopy (SEM) and energy dispersive X-ray (EDX). A Zeiss EVO LS 25 microscope equipped with an Oxford INCA Xact X-ray detector was used for the SEM and EDX analyses. High resolution SEM measurements were carried out with a field emission SEM (Jeol JSM 7500F). X-ray photoelectron spectroscopy (XPS) data were obtained using a ThermoScientific Theta Probe System with $\mathrm{Al}-\mathrm{K}_{\alpha}$ radiation (photon energy=1486.6 eV). Where necessary, surface contamination was eliminated by the use of an ion sputtering gun. The $\mathrm{Zr} 3 \mathrm{~d}, \mathrm{O} 1 \mathrm{~s}$, and $\mathrm{C} 1 \mathrm{~s}$ spectra were collected. All data were aligned against the $\mathrm{C} 1 \mathrm{~s}$ peak at the binding energy of $284.6 \mathrm{eV}$.

The resistive switching behaviour of the bilayer $\mathrm{ZrO}_{2-x} / \mathrm{ZrO}_{2}$ structure was investigated by fabricating memory devices. A $200 \mathrm{~nm}$ thick TiN film was reactively sputtered (Ti target in a $\mathrm{N}_{2}$ atmosphere) onto the $\mathrm{SiO}_{2}$ layer to form the bottom electrode. This was followed by reactive sputtering of a second $\mathrm{SiO}_{2}$ layer ( $\mathrm{Si}$ target in an $\mathrm{O}_{2}$ atmosphere). This layer of $\mathrm{SiO}_{2}$ was patterned to form active device areas by photolithography and reactive ion etch. After that, a $20 \mathrm{~nm} \mathrm{ZrO}$ layer and a $5 \mathrm{~nm} \mathrm{ZrO}_{2-x}$ layers were deposited subsequently to form the switching layer. Finally, a $200 \mathrm{~nm}$ TiN layer was sputtered and patterned to form the top electrode. All electrical measurements were performed with a Keithley 4200 semiconductor characterization system. During the measurements, the programming voltage bias was applied to the top electrode, while keeping the bottom electrode grounded. The probe/point contacts to the top and bottom electrodes of the devices were realized through a pair of Wentworth probe needles, using a Wentworth laboratories AVT 702 semi-automatic prober.

\section{Results and discussion}

Compositional characterisations were firstly carried out on the as-deposited $\mathrm{ZrO}_{\mathrm{x}}$ films with $\mathrm{O}_{2}$ flow rate ranging from $6 \mathrm{sccm}$ to $20 \mathrm{sccm}$ by EDX. Films with a large thickness of $1 \mu \mathrm{m}$ were deposited directly onto $\mathrm{Si}$ wafers to minimise the influence from the substrate. EDX spectra of all 8 films are displayed in Figure 1a where only $\mathrm{Zr}$ and $\mathrm{O}$ peaks are detected. For direct comparison, all spectra were nominalised against the $\mathrm{Zr}$ peak at $2.042 \mathrm{keV}$. An increase of the $\mathrm{O}$ peak $(0.525 \mathrm{keV})$ intensity is observed as the $\mathrm{O}_{2}$ flow rate changes from $6 \mathrm{sccm}$ to $12 \mathrm{sccm}$, indicating an increasing $\mathrm{O}$ composition in the resultant films. Quantitative EDX results reveal 
that stoichiometric $\mathrm{ZrO}_{2}$ films are deposited when the $\mathrm{O}_{2}$ flow rate reached $12 \mathrm{sccm}$ (shown in Figure $1 \mathrm{~b}$ ). For $\mathrm{ZrO}_{2-x}$ films deposited under flow rates of $6 \mathrm{sccm}, 8 \mathrm{sccm}$ and $10 \mathrm{sccm}$, substoichiometric films with compositions of $\mathrm{ZrO}_{1.2}, \mathrm{ZrO}_{1.5}$ and $\mathrm{ZrO}_{1.8}$, respectively, are obtained (see Figure S2 in the supplementary for the SEM cross-section view of these films). The O:Zr ratio saturates when more $\mathrm{O}_{2}$ was introduced into the chamber and stoichiometric $\mathrm{ZrO}_{2}$ films were obtained.
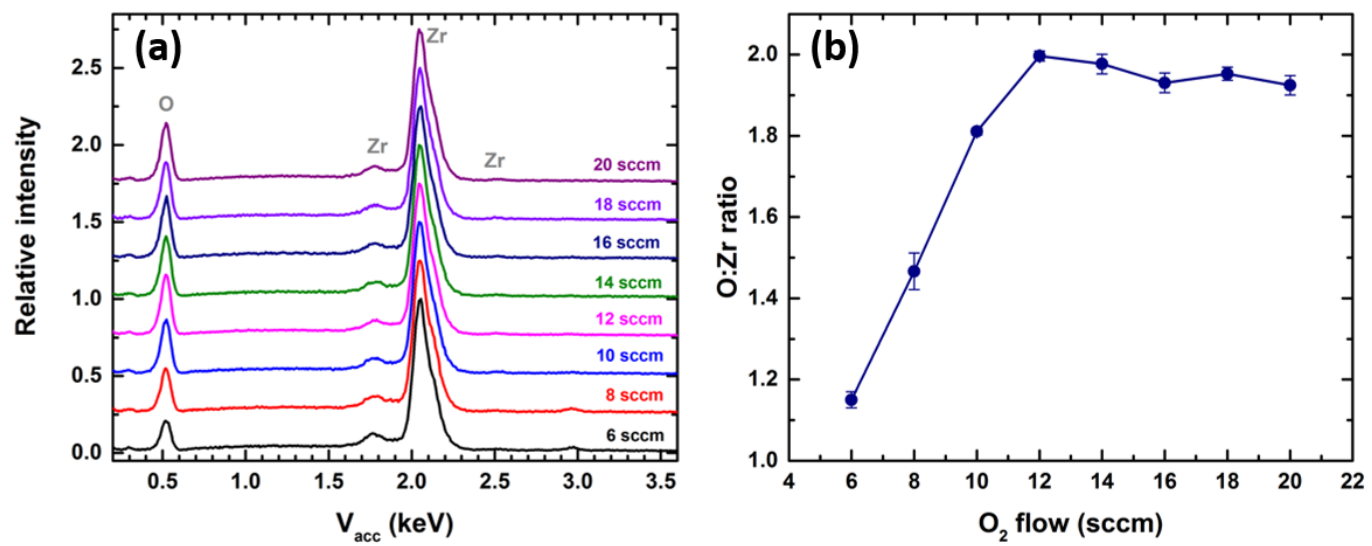

Figure 1. (a) EDX spectra and (b) quantitative O:Zr ratio of the as-deposited $\mathrm{ZrO}_{\mathrm{x}}$ films with different $\mathrm{O}_{2}$ flow rates from $6 \mathrm{sccm}$ to $20 \mathrm{sccm}$.

The resistive switching properties of a bilayer TiN/ZrO $\mathrm{Zr.8}_{1.8} / \mathrm{ZrO}_{2} / \mathrm{TiN}$ memory device was firstly investigated. The device schematic is shown in Figure 2a. The resistance of a pristine cell is usually higher than $10^{10} \Omega$, and an electroforming process is required to change the cell into a comparatively conductive state. Here, a negative bias of $-6 \mathrm{~V}$ is applied to the cell with a current compliance (CC) at $1 \mathrm{~mA}$, as shown in Figure $2 \mathrm{~b}$. An abrupt increase of current is observed at $c a$. $-5.4 \mathrm{~V}$ and the device is transformed into low resistance state (LRS) of $c a .200 \Omega$. A positive DC sweep of $5 \mathrm{~V}$ is then applied to the cell during which a negative differential resistance effect takes place and the cell was reset into a high resistance state (HRS) as shown in Figure 2c. The cell is subsequently switched back into the LRS by a negative bias with a CC of $20 \mathrm{~mA}$, showing a typical bipolar switching behaviour. We hereby refer this as the negative bipolar mode. The endurance characteristics for the device in this negative SET mode is shown in Figure $2 \mathrm{~d}$ where the device is switched ON and OFF for 50 times with resistance values measured at $0.1 \mathrm{~V}$. The device demonstrates a reproducible switching behaviour with sufficient ON/OFF ratio of $c a$. 20 . 

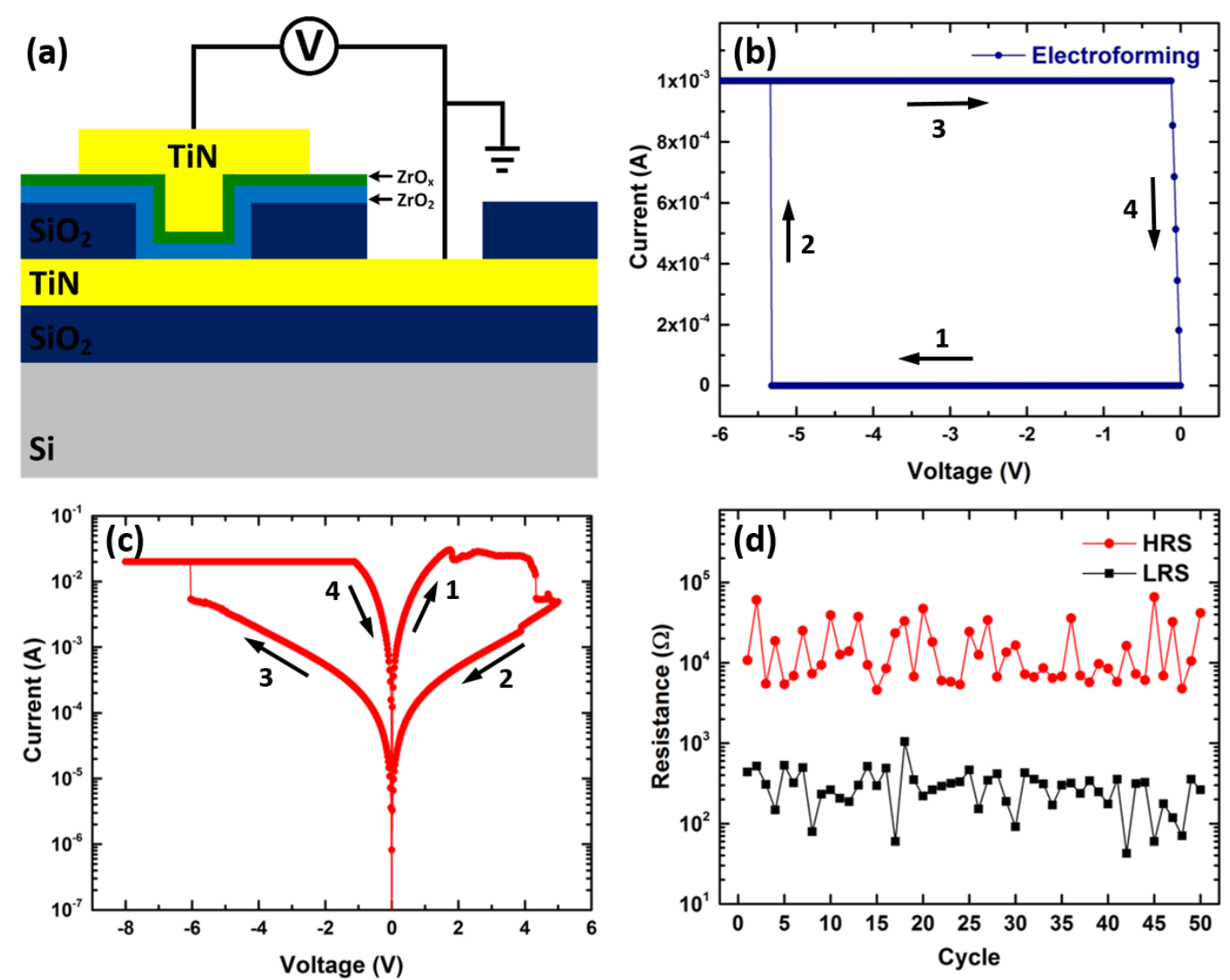

Figure 2. (a) Structure of the $\mathrm{TiN} / \mathrm{ZrO}_{1.8} / \mathrm{ZrO}_{2} / \mathrm{TiN}$ resistive memory device. Throughout the electrical measurements, bias voltages were applied on the top TiN electrode while the bottom TiN electrode was grounded. (b) Electroforming process of the bilayer $\mathrm{TiN} / \mathrm{ZrO}_{1.8} / \mathrm{ZrO}_{2} / \mathrm{TiN}$ device with a $\mathrm{CC}$ of $1 \mathrm{~mA}$. (c) $I$ - $V$ characteristics of the device after forming. (d) The endurance characteristics of the bilayer device.

When an identical bilayer device $\left(\mathrm{TiN} / \mathrm{ZrO}_{1.8} / \mathrm{ZrO}_{2} / \mathrm{TiN}\right)$ is initialized with a lower forming CC of $1 \mu \mathrm{A}$, a similar forming voltage is observed with a much higher resistance state of $c a .40 \mathrm{k} \Omega$ (shown in Figure $3 \mathrm{a}$ ). The $I-V$ characteristics of the device after such forming are very different as demonstrated in Figure $3 \mathrm{~b}$. When a positive bias was applied on the device, an abruptly decrease of the resistance to a LRS $(c a .1 \mathrm{k} \Omega)$ is observed when the bias reaches $0.8 \mathrm{~V}(\mathrm{CC}=1$ $\mathrm{mA}$ ). When a negative bias is subsequently applied, the resistance comes back to the HRS, showing a bipolar switching behaviour with reversed polarity. We hereby refer it as the positive bipolar mode. It is worth mentioning that a CC is required for the RESET process (reason will be discussed later) and the $V_{R E S E T}$ for this mode is defined at the point where the current is just below the CC in process " 4 " of Figure $3 b$. Figure $3 c$ displays cycling endurance characteristics where reversible and reproducible resistive switching property is demonstrated for more than 100 cycles with an ON/OFF ratio of $c a$. 40. The stable retention performance is also confirmed at room temperature for $10^{4} \mathrm{~s}$, as shown in Figure $3 \mathrm{~d}$. 

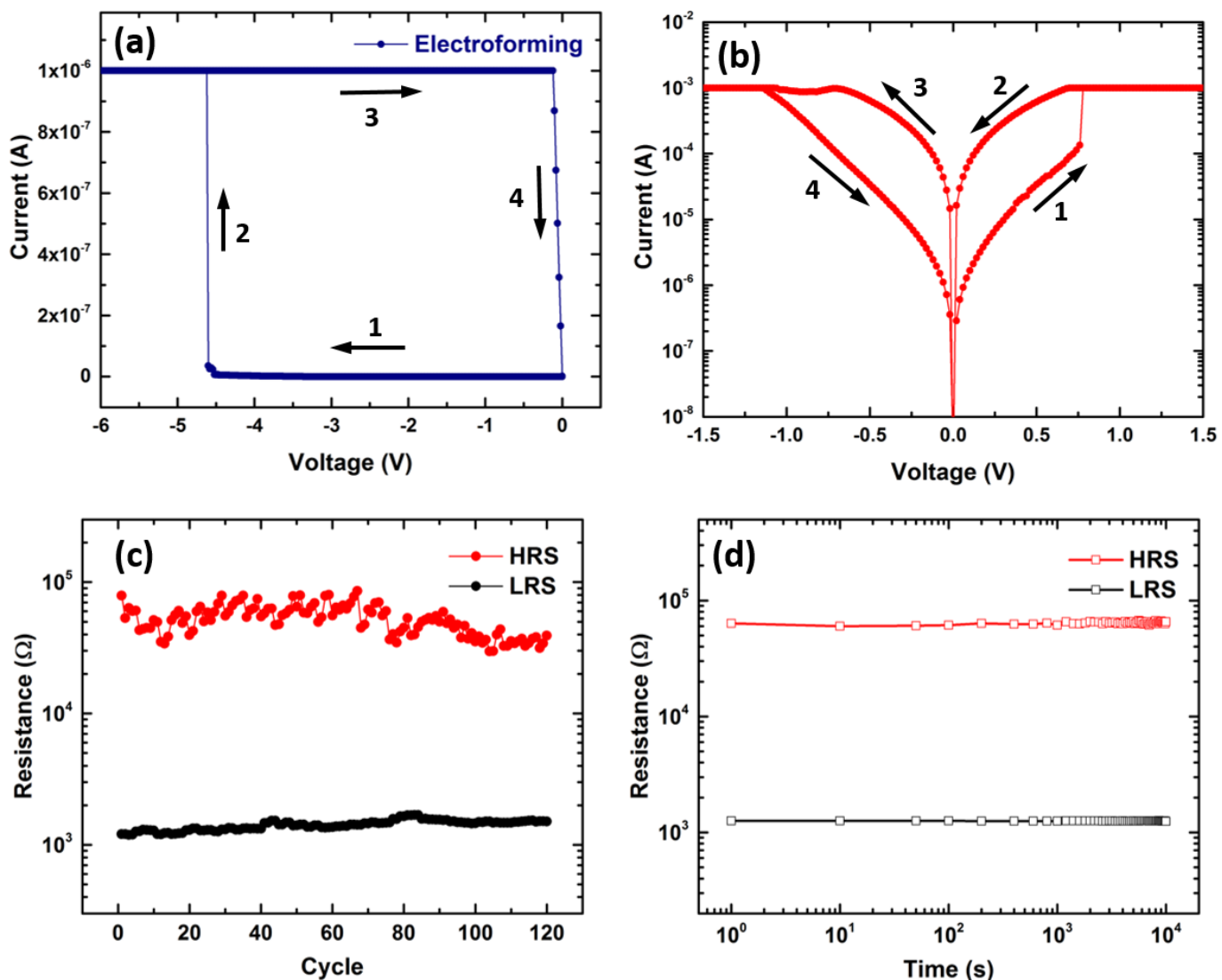

Figure 3. (a) Electroforming process of the bilayer $\mathrm{TiN} / \mathrm{ZrO}_{1.8} / \mathrm{ZrO}_{2} / \mathrm{TiN}$ device with a $\mathrm{CC}$ of $1 \mu \mathrm{A}$. (b) $I-V$ characteristics of the device for after forming. The endurance (c) and the retention (d) characteristics of the bilayer device.

Apart from the reversed switching polarity, the positive bipolar mode also demonstrates a much more improved performance. Figure 4 shows the cumulative probability distribution of the switching parameters of the two switching modes from measurements on 20 devices. The positive bipolar mode has a lower $\mathrm{V}_{\text {SET }}$ and $\mathrm{V}_{\text {RESET }}$ with much narrower distributions (shown in Figure 4a). The coefficients of the variation $(\sigma / \mu, \sigma$ is the standard deviation and $\mu$ is the mean value) of $V_{S E T}$ and $V_{R E S E T}$ were found to be reduced from $10.7 \%$ and $14.6 \%$ in the negative bipolar mode to $4.7 \%$ and $3.8 \%$ in the positive bipolar mode. The high uniformities of both $V_{S E T}$ and $V_{R E S E T}$ are essential for the large-scale RRAM applications. Furthermore, it is also clear that the both LRS and HRS are distributed more uniformly under the positive SET mode as shown in Figure $4 b$.
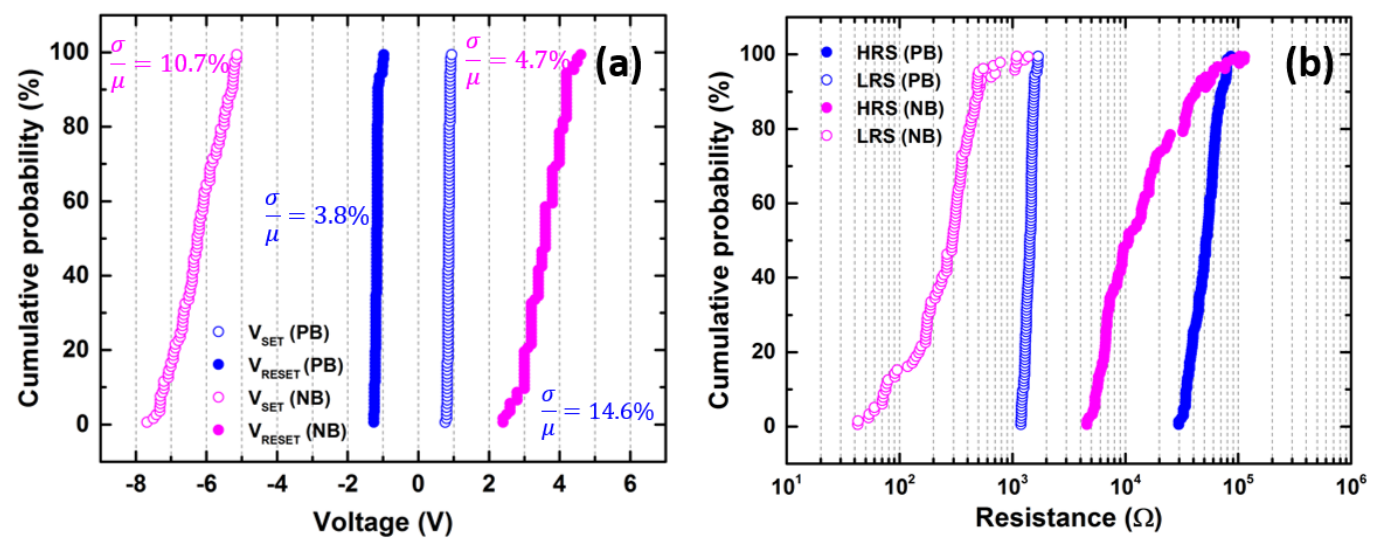

Figure 4. Cumulative probability graphs of (a) $V_{S E T}$ and $V_{R E S E T}$ and (b) HRS and LRS for both the positive bipolar (PB) mode and negative bipolar (NB) mode on the bilayer TiN/ZrO ${ }_{1.8} / \mathrm{ZrO}_{2} / \mathrm{TiN}$ device. 
In order to better understand the mechanism of this bilayer device, memory cells containing only a single $\mathrm{ZrO}_{2}$ layer were fabricated and tested (shown in Figure S2). At an electroforming $\mathrm{CC}$ of $1 \mathrm{~mA}$, only the negative bipolar switching mode was observed, which was similar to that of the bilayer device. The logarithmic $I-V$ curve plots and linear fittings of the SET process in this negative bipolar mode for both the single layer and bilayer device are presented in Figure 5 and show near identical behaviour. The SET curve suggests the current is governed by the space charge limited current (SCLC) model with traps, which consist of three regions: Ohmic region $(I \propto V)$, the Child's law region $\left(I \propto V^{2}\right)$ and the steep current increase region, as demonstrated by the different slopes of the linear fits [11,26,27]. The Ohmic conduction behaviour at low voltage could possibly be due to the fact that the density of thermally generated electrons inside the films is larger than the injected electrons from the electrode. The slope then changes to $c a .2$ under larger voltage values, indicating the domination of space charge limited current. In this circumstance, the injected charge carriers dominate the conduction while the thermally generated charge concentration is negligible. After that, the formation of conducting paths and trapping of the injected charges start to occur, and an abrupt increase of current is observed. Eventually, the voltage reaches $V_{S E T}$ and the device is switched to LRS, which is featured by an Ohmic conduction at low voltage with the addition of a quadratic term at higher voltage, suggesting the existence of trapped electrons in the conductive paths. Similar observations of this SCLC mechanism were also reported in other $\mathrm{ZrO}_{2}$-based resistive memory devices $[11,17,28]$. This suggests that the additional $\mathrm{ZrO}_{1.8}$ layer does not affect the device switching behaviour when the forming CC of $1 \mathrm{~mA}$ was applied.
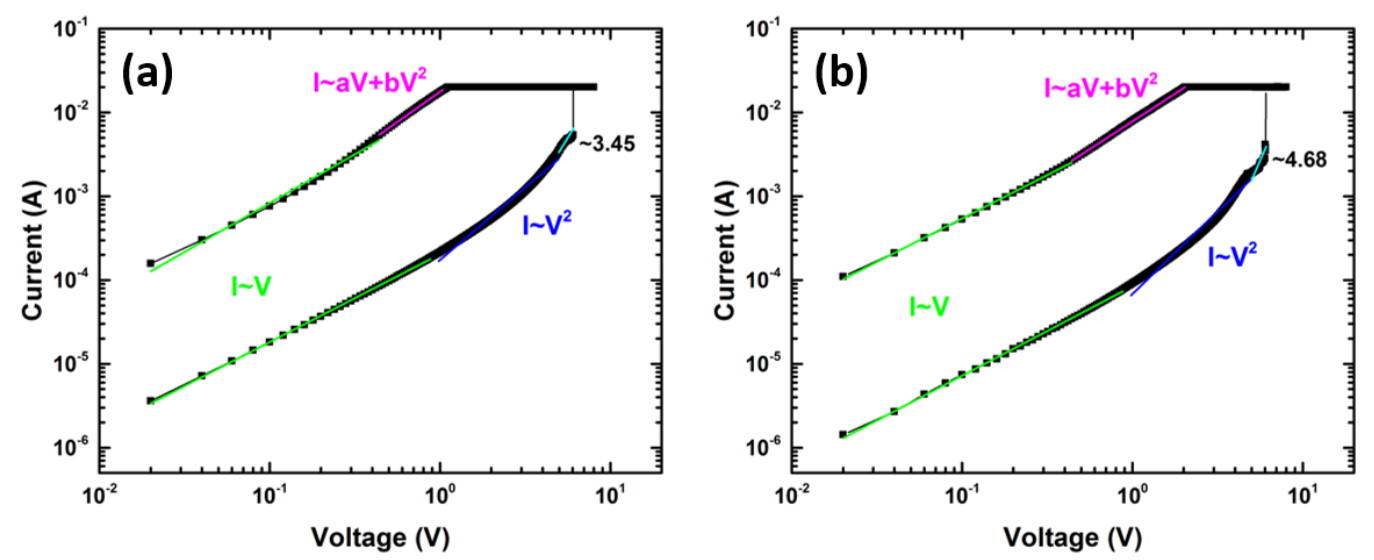

Figure 5. $I-V$ characteristics and the SET process $I-V$ curves in double-logarithmic plots of the (a) single layer $\mathrm{TiN} / \mathrm{ZrO}_{2} / \mathrm{TiN}$ device and (b) bilayer TiN/ZrO ${ }_{1.8} / \mathrm{ZrO}_{2} / \mathrm{TiN}$ device formed with a CC of $1 \mathrm{~mA}$.

A lower $\mathrm{CC}$ of $1 \mu \mathrm{A}$ was also applied to initialize the single $\mathrm{ZrO}_{2}$ layer device but this did not result in a positive bipolar switching. Only negative bipolar mode was observed with $I-V$ characteristics similar to devices electroformed under $1 \mathrm{~mA} \mathrm{CC}$ (shown in Figure S2c). This observation clearly indicates that the positive bipolar mode is a unique of the bilayer device. To shed light on the mechanism of this switching behaviour, bilayer structures of $\mathrm{ZrO}_{1.2} / \mathrm{ZrO}_{2}$ and $\mathrm{ZrO}_{1.5} / \mathrm{ZrO}_{2}$ were compared with the $\mathrm{ZrO}_{1.8} / \mathrm{ZrO}_{2}$ structure. $I-V$ characteristics of these three type of devices after electroforming $(\mathrm{CC}=1 \mu \mathrm{A})$ are shown in Figure 6a-6c. The positive bipolar mode was observed on all three type of devices while the switching performance is strongly affected by the top $\mathrm{ZrO}_{2-\mathrm{x}}$ used. While the $\mathrm{ZrO}_{1.8} / \mathrm{ZrO}_{2}$ bilayer device demonstrates good resistive switching behaviour (Figure $6 \mathrm{c}$ ), $\mathrm{ZrO}_{1.2} / \mathrm{ZrO}_{2}$ and $\mathrm{ZrO}_{1.5} / \mathrm{ZrO}_{2}$ bilayer devices display relatively poor switching performances (Figure 6a-6b). It is observed that the HRSs in those two types of devices are lower than that of the $\mathrm{ZrO}_{1.8} / \mathrm{ZrO}_{2}$ bilayer device, and once being switched ON, they cannot be fully reset back to the original HRSs. Despite the different switching performances, the three types of devices all demonstrate similar current conduction mechanism which are in good agreement with the SCLC model as shown in Figure 6d-6f. 

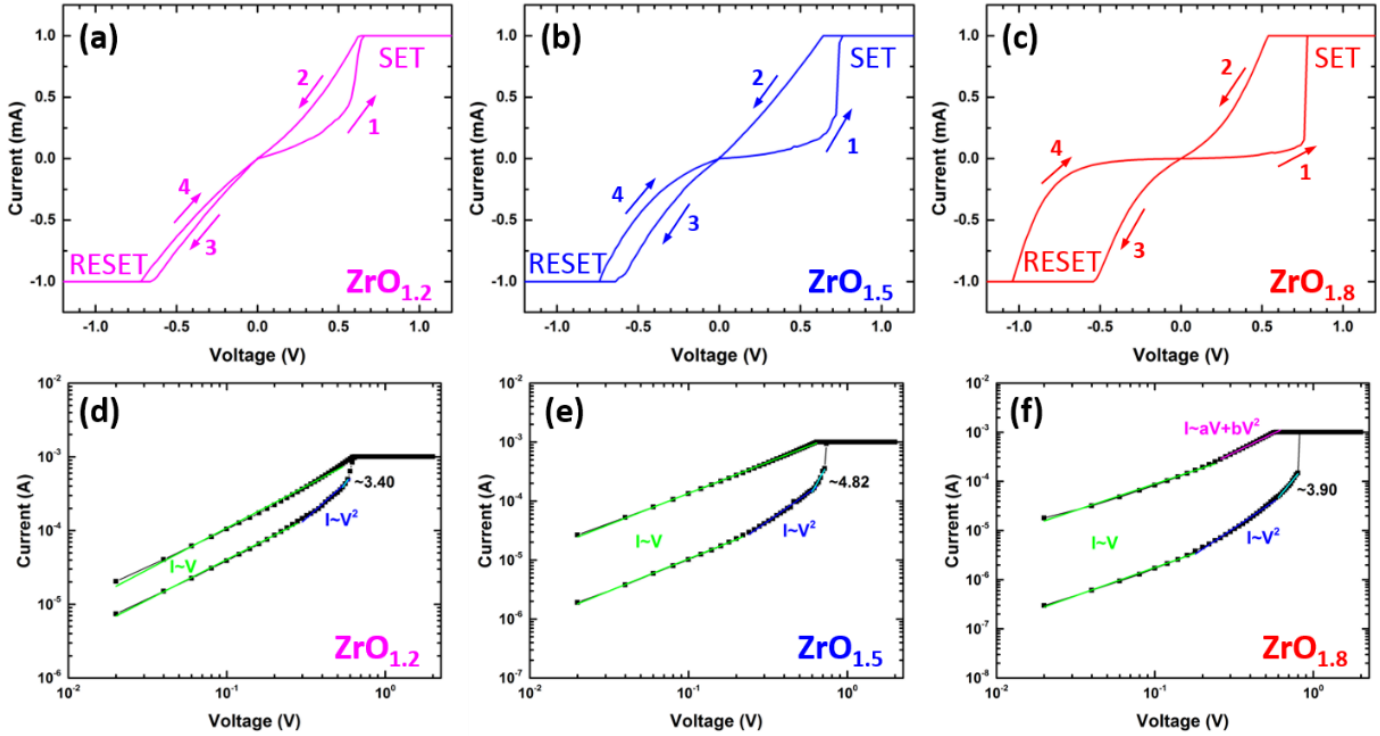

Figure 6. $I-V$ characteristics and the SET process $I-V$ curves in double-logarithmic plots of the bilayer $\mathrm{TiN} / \mathrm{ZrO}_{2}$ ${ }_{x} / \mathrm{ZrO}_{2} / \mathrm{TiN}$ devices with the composition of the top $\mathrm{ZrO}_{2-\mathrm{x}}$ layer being (a, d) $\mathrm{ZrO}_{1.2}$, (b, e) $\mathrm{ZrO}_{1.5}$ and (c, f) $\mathrm{ZrO}_{1.8}$ electroformed under a CC of $1 \mu \mathrm{A}$.

XPS analysis was performed to investigate the oxidation states within the $\mathrm{ZrO}_{1.2}, \mathrm{ZrO}_{1.5}, \mathrm{ZrO}_{1.8}$ and $\mathrm{ZrO}_{2.0}$ films and the $\mathrm{Zr} 3 \mathrm{~d}$ core level spectra are presented in Figure 7. The $\mathrm{Zr} 3 \mathrm{~d}$ spectrum of the $\mathrm{ZrO}_{1.2}$ (Figure 7a-7b) exhibits a pronounced doublet with main peak at $c a .178 .4 \mathrm{eV}$, which can be ascribed to the metallic zirconium $\left(\mathrm{Zr}^{0}\right)$ pairs. The substantial amount of this metallic $\mathrm{Zr}^{0}$ bonding is possibly due to the low oxygen concentration in the $\mathrm{ZrO}_{1.2}$ film. The intensity of the stoichiometric $\mathrm{ZrO}_{2}$ related $\mathrm{Zr}^{4+}$ oxide doublet $(c a .183 .4 \mathrm{eV})$, on the other hand, are detected to be significantly lower. This also implies the existence of $\mathrm{Zr}$ sub-valence states (valences states between $\mathrm{Zr}^{0}$ and $\mathrm{Zr}^{4+}$ ) which is due to the presence of nonstoichiometric $\mathrm{Zr}$ oxides [29]. Although a conclusive proof of the number and nature of these states is yet to be reached, numerous reports have presented similar results [29-32]. In this work, two Zr suboxide states marked as $\mathrm{Zr}^{\mathrm{a}+}$ and $\mathrm{Zr}^{\mathrm{b}+}$ are adopted in fitting. The intensity of these two doublets (ca. $180.0 \mathrm{eV}$ and $181.8 \mathrm{eV}$ ) increases with the increasing oxygen concentration from $\mathrm{ZrO}_{1.2}$ to $\mathrm{ZrO}_{1.5}$ film. This is accompanied by the decrease of $\mathrm{Zr}^{0}$ bonding while the amount of $\mathrm{Zr}^{4+}$ component remains low (Figure $7 \mathrm{~b}$ ). This suggests the metallic zirconium has mostly been oxidized into sub-oxide states at this stage. When the oxygen concentration reaches $\mathrm{ZrO}_{1.8}$, all metallic zirconium has been transferred into oxide forms (Figure 7c) and better switching behaviour of the bilayer device is observed in Figure 6c. This implies that the presence of the metallic zirconium component in both $\mathrm{ZrO}_{1.2}$ and $\mathrm{ZrO}_{1.5}$ layers largely deteriorated the switching performance of the bilayer device. The amount of stoichiometric $\mathrm{Zr}^{4+}$ state within the $\mathrm{ZrO}_{1.8}$ film has also started to increase significantly (Figure 7c) and become dominant in the $\mathrm{ZrO}_{2}$ film (Figure 7d). 

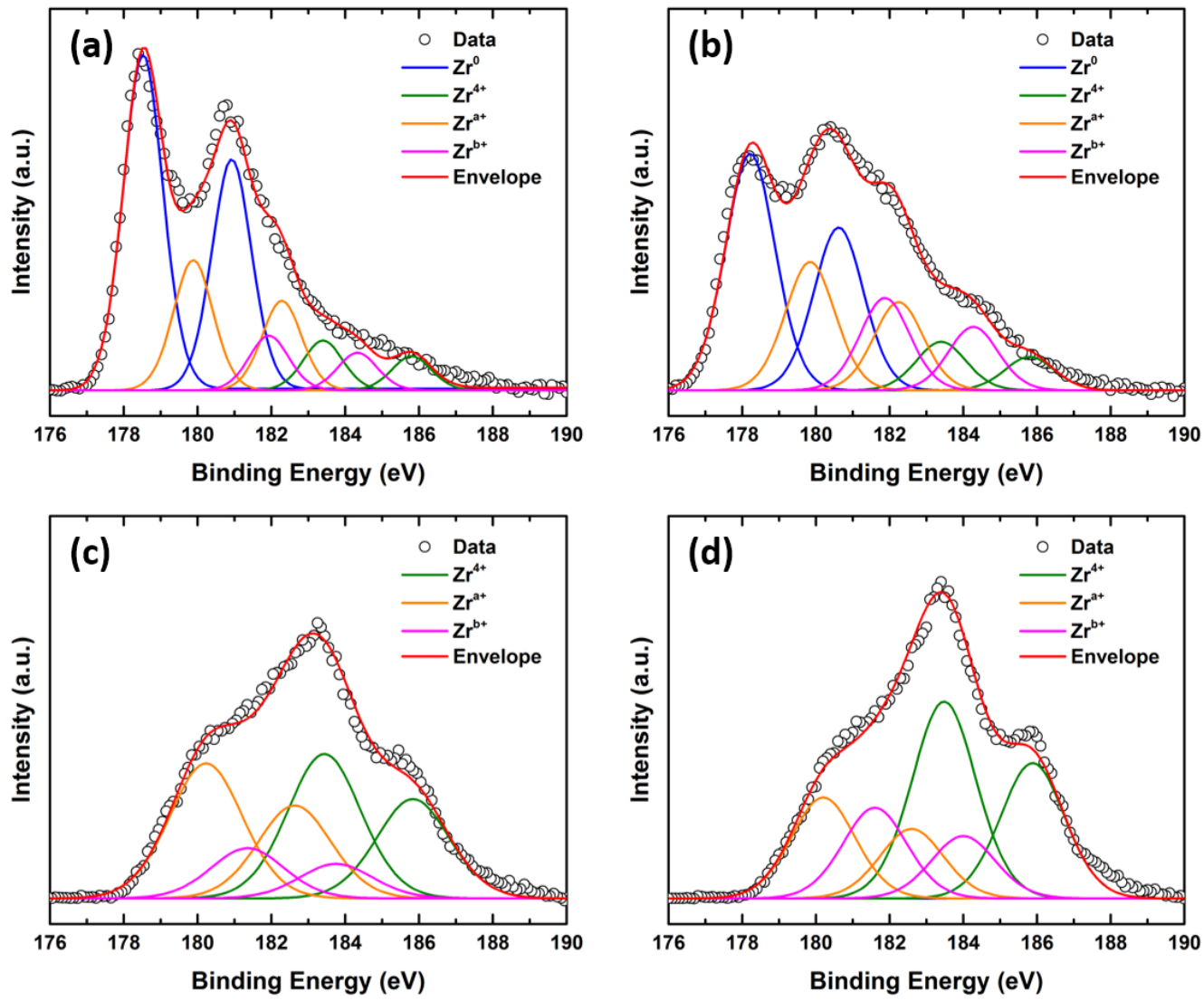

Figure 7. XPS spectra and their Gaussian fittings of the $\mathrm{Zr} 3 \mathrm{~d}$ for (a) $\mathrm{ZrO}_{1.2}$, (b) $\mathrm{ZrO}_{1.5}$, (c) $\mathrm{ZrO}_{1.8}$ and $\mathrm{ZrO}_{2.0}$ films, respectively. Doublets are fitted with the spin-orbit splitting between the $3 \mathrm{~d}_{5 / 2}$ and $3 \mathrm{~d}_{3 / 2}$ peaks fixed to be $2.4 \mathrm{eV}$.

Based on conduction mechanism analysis and the structure measurement discussed above, the possible scenarios of the switching behaviours are proposed. For a single layer $\mathrm{TiN} / \mathrm{ZrO} / \mathrm{TiN}$ device, a thin TiON layer is likely formed after the deposition of $\mathrm{ZrO}_{2}$ at the $\mathrm{ZrO}_{2} / \mathrm{TiN}$ interface (Figure 8a). The formation of this TiON layer on the bottom interface is due to the fact that $\mathrm{ZrO}_{2}$ layer was immediately sputtered after the sputtering of bottom TiN electrode. In the first few rotations, the $\mathrm{O}_{2}$ plasma has likely reacted with the TiN surface to form a TiON layer as the $\mathrm{ZrO}_{2}$ layer is still very thin $(<1 \mathrm{~nm})$. This interfacial TiON plays a crucial role in the bipolar behaviour as it serves as an oxygen reservoir [33,34]. During the electroforming process, a large voltage drop is across the $\mathrm{ZrO}_{2}$ layer, where the creation of oxygen vacancies will be triggered by the high electric field. Conductive filament made of oxygen vacancies are formed during the electroforming process and the oxygen ions are forced to migrate into the TiON reservoir under a negative voltage (Figure 8b). The device is hence in LRS due to the high conductivity of the filament. At the RESET process, the oxygen ions in the reservoir are pushed back to by the opposite RESET bias and react with the vacancies near the electrode. The filament is broken and the device is switched from LRS back to HRS (Figure 8c). A negative voltage is then required to re-connect the filament and SET the device back to LRS (Figure 8d). Similar bipolar switching behaviours was also observed in $\mathrm{HfO}_{2}$ and $\mathrm{ZnO}$ for the same device structure $[9,35]$. 


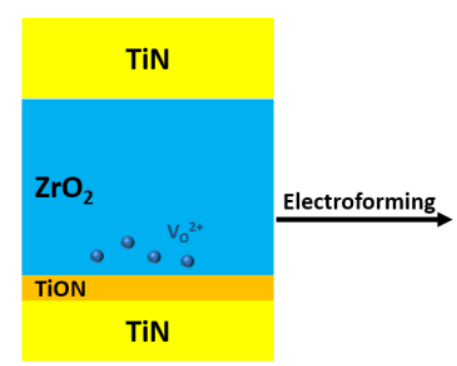

(a)

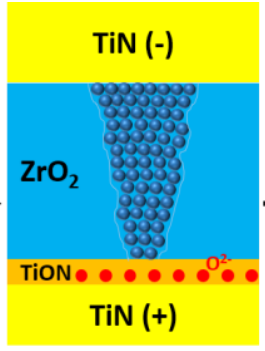

(b)

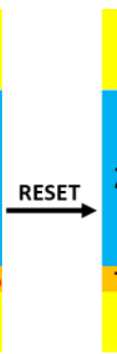

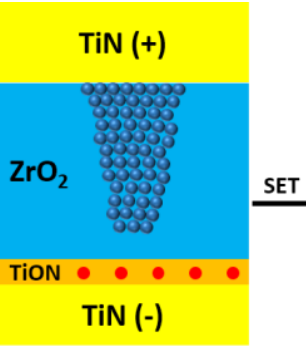

(c)

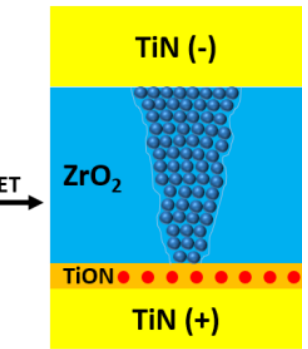

(d)

Figure 8. Schematic illustration for bipolar resistive switching mechanism for the single layer $\mathrm{TiN} / \mathrm{ZrO}_{2} / \mathrm{TiN}$ device; Blue dots are oxygen vacancies and red dots are oxygen ions.

The switching models for the bilayer $\mathrm{TiN} / \mathrm{ZrO}_{1.8} / \mathrm{ZrO}_{2} / \mathrm{TiN}$ devices are illustrated in Figure 9. In this circumstance, the voltage drop during the electroforming process is mainly across the bulk $\mathrm{ZrO}_{2}$ layer where the creation of oxygen vacancies takes place. In the case of a low forming $\mathrm{CC}(1 \mu \mathrm{A})$, a weak filament is formed in the $\mathrm{ZrO}_{2}$ layer (Figure 9b), leading to an abrupt increase of current as shown in Figure 3a. However, as the filament is not continuous within the $\mathrm{ZrO}_{2-\mathrm{x}}$ layer, the electroformed device still exhibits a relatively high resistance, During the subsequent SET process, the positive voltage drop is now mainly across the $\mathrm{ZrO}_{2-\mathrm{x}}$ layer, where oxygen vacancies would be quickly created to form the conductive channel. The oxygen ions are attracted to the $\mathrm{ZrO}_{2-x} / \mathrm{TiN}$ interface which consequently serves as an oxygen reservoir and the device is transferred into LRS (Figure 9c). When a negative RESET voltage is applied, oxygen ions in the interface migrate into the $\mathrm{ZrO}_{2-x}$ layer and react with the vacancies. The filament is dissolved and ruptured, and the device switches back to HRS (Figure 9d). It should be noted that this confinement of the filament size within the $\mathrm{ZrO}_{2-\mathrm{x}}$ layer contributes to the improved switching performances observed in the positive bipolar mode. The degrading switching performance observed for the $\mathrm{ZrO}_{1.2} / \mathrm{ZrO}_{2}$ and $\mathrm{ZrO}_{1.5} / \mathrm{ZrO}_{2}$ bilayer devices could be explained by the high concentration of oxygen vacancies originate in those two films which lead to the formation of a much stronger filament. This filament is difficult to be dissolved or ruptured due to the insufficient oxygen ions. Under a high forming CC (1 mA), that a strong filament is formed across both $\mathrm{ZrO}_{2}$ and $\mathrm{ZrO}_{2-\mathrm{x}}$ layers due to the intense current flows inside the layers and the device is transferred directly into LRS (Figure 9e). The subsequent switching scenario is similar to that of the single layer $\mathrm{TiN} / \mathrm{ZrO}_{2} / \mathrm{TiN}$ device, as shown in Figure $9 \mathrm{f}-9 \mathrm{~g}$. It is worth mentioning that the polarity of forming voltage does not affect this switching behaviour for both single layer and bi-layer devices as similar results were also observed under positive forming voltages. The filament formed within the $\mathrm{ZrO}_{2-\mathrm{x}}$ layer is non-reversible in this case, possibly due to the excess amount of oxygen vacancies in the filament as a result of a high current [1]. This also explains the requirement of a $1 \mathrm{~mA} \mathrm{CC}$ for both SET and RESET processes in the positive bipolar mode, to avoid the irreversible formation of this strong filament. Higher CC was applied during the RESET process in this mode and the device was observed to be switched into a lower resistance state and subsequently demonstrate bipolar switching behaviour similar to Figure 2c, indicating the formation of strong filament (shown in Figure S3). 


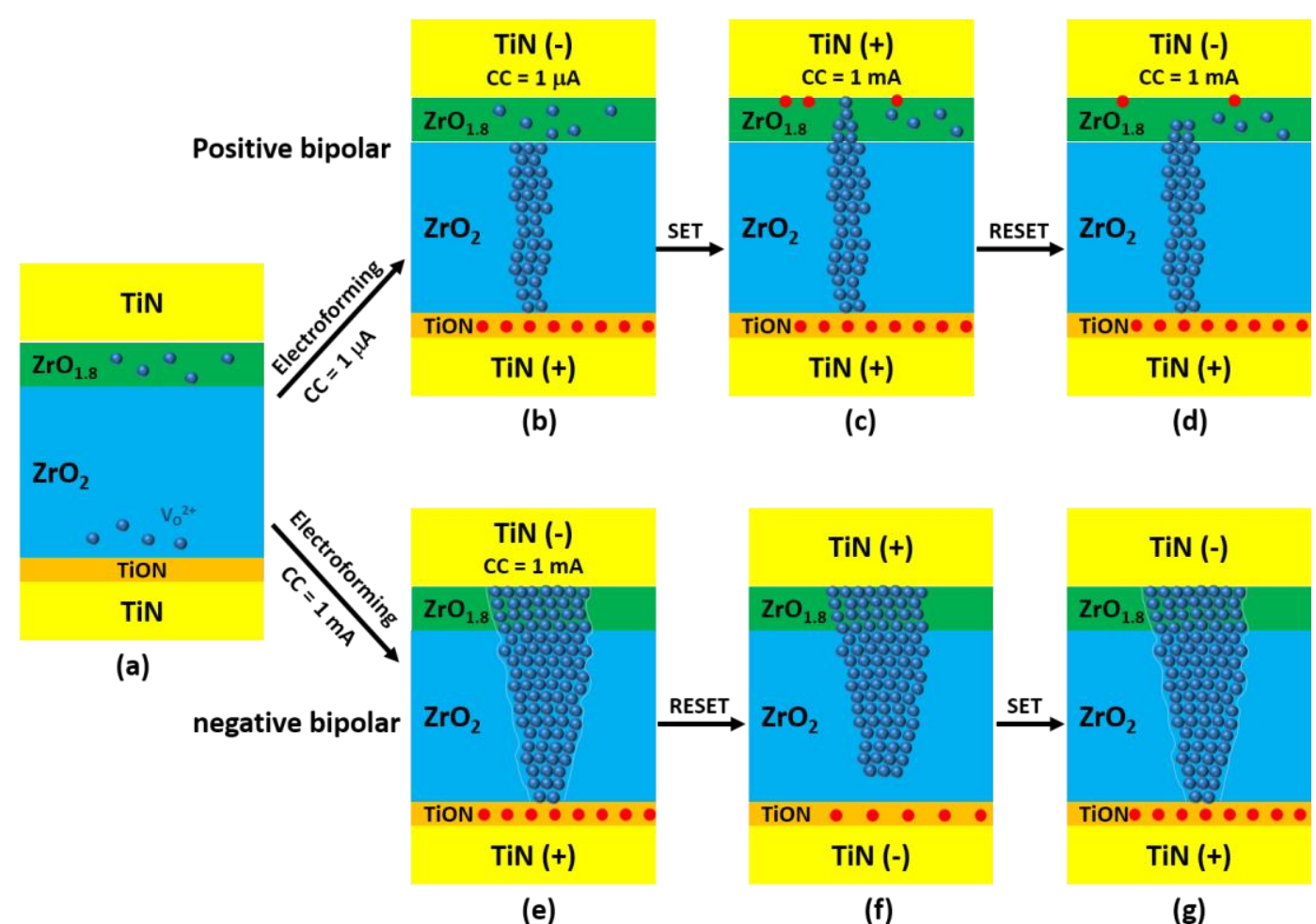

Figure 9. Schematic illustrations for $1 \mu \mathrm{A}$ and $1 \mathrm{~mA} \mathrm{CC}$ forming and the subsequent switching mechanisms of the bilayer TiN/ZrO ${ }_{1.8} / \mathrm{ZrO}_{2} / \mathrm{TiN}$ device. Red dots represent oxygen ions and blue dots represent oxygen vacancies.

\section{Conclusion}

In conclusion, distinctive programmable resistive switching behaviours have been demonstrated in bilayer $\mathrm{TiN} / \mathrm{ZrO}_{2-x} / \mathrm{ZrO}_{2} / \mathrm{TiN}$ devices. Both positive and negative bipolar switching can be achieved within the same device by applying different current compliance during the electroforming process. The positive bipolar mode demonstrates enhanced switching performance with low switching voltage, narrow switching voltage distribution and good cycling endurance. It has been revealed that oxygen concentration level in the $\mathrm{ZrO}_{2-\mathrm{x}}$ layer plays an important role in this positive bipolar mode whereas an excess of oxygen vacancies would lead to the deterioration of the switching performance. The formation and dissolution of the oxygen vacancy filaments have been used to explain both bipolar switching behaviours and the improved properties in the positive bipolar mode are attributed to the confined oxygen vacancy filament size within $\mathrm{ZrO}_{2-x}$ layer. The paper clearly contributes towards the future development of switching memory and particularly the advantages of using a bilayer of stoichiometric and sub-stoichiometric metal oxide with better switching performances.

\section{Acknowledgements}

We thank the Engineering and Physical Sciences Research Council (EPSRC) for support (EP/I010890/1) and for a Doctoral Prize (R.H. EP/509015FP/1). All data can be found on dataset doi: 10.5258/SOTON/xxxxx.

\section{References}

[1] Wong H-S P, Lee H-Y, Yu S, Chen Y-S, Wu Y, Chen P-S, Lee B, Chen F T and Tsai M-J 2012 Metal-Oxide RRAM Proc. IEEE 100 1951-1970

[2] Yang J J, Pickett M D, Li X, Ohlberg D a a, Stewart D R and Williams R S 2008 Memristive switching mechanism for metal/oxide/metal nanodevices. Nat.

Nanotechnol. 3 429-433 
[3] Kwon D-H, Kim K M, Jang J H, Jeon J M, Lee M H, Kim G H, Li X-S, Park G-S, Lee B, Han S, Kim M and Hwang C S 2010 Atomic structure of conducting nanofilaments in $\mathrm{TiO}_{2}$ resistive switching memory. Nat. Nanotechnol. 5 148-53

[4] Kim Y M and Lee J S 2008 Reproducible resistance switching characteristics of hafnium oxide-based nonvolatile memory devices J. Appl. Phys. 104114115

[5] Morgan K A, Huang R, Potter K, Shaw C, Redman-White W and De Groot C H 2014 Total Dose Hardness of TiN/HfO $/$ TiN Resistive Random Access Memory IEEE Trans. Nucl. Sci. 61 2991-2996

[6] Yang Y, Zhang X, Gao M, Zeng F, Zhou W, Xie S and Pan F 2011 Nonvolatile resistive switching in single crystalline ZnO nanowires. Nanoscale 3 1917-21

[7] Yalishev V S, Yuldashev S U, Kim Y S and Park B H 2012 The role of zinc vacancies in bipolar resistance switching of $\mathrm{Ag} / \mathrm{ZnO} / \mathrm{Pt}$ memory structures. Nanotechnology 23375201

[8] Xue W H, Xiao W, Shang J, Chen X X, Zhu X J, Pan L, Tan H W, Zhang W B, Ji Z H, Liu G, Xu X-H, Ding J and Li R-W 2014 Intrinsic and interfacial effect of electrode metals on the resistive switching behaviors of zinc oxide films. Nanotechnology 25425204

[9] Huang R, Sun K, Kiang K S, Morgan K A and de Groot C H 2016 Forming-free resistive switching of tunable $\mathrm{ZnO}$ films grown by atomic layer deposition Microelectron. Eng. 161 7-12

[10] Tsai T-L, Ho T-H and Tseng T-Y 2015 Unipolar resistive switching behaviors and mechanisms in an annealed $\mathrm{Ni} / \mathrm{ZrO}_{2} / \mathrm{TaN}$ memory device J. Phys. D. Appl. Phys. 48 35108

[11] Du G, Li T, Wang C, Fang B, Zhang B and Zeng Z 2015 Engineering of forming-free resistive switching characteristics in $\mathrm{ZrO}_{2}$ films J. Phys. D. Appl. Phys. 48225301

[12] Lin C Y, Wu C Y, Wu C Y, Lin C C and Tseng T Y 2007 Memory effect of RF sputtered $\mathrm{ZrO}_{2}$ thin films Thin Solid Films 516 444-448

[13] Wang S-Y, Lee D-Y, Huang T-Y, Wu J-W and Tseng T-Y 2010 Controllable oxygen vacancies to enhance resistive switching performance in a $\mathrm{ZrO}_{2}$-based $\mathrm{RRAM}$ with embedded Mo layer. Nanotechnology 21495201

[14] Lin C-L and Lin T-Y 2016 Superior unipolar resistive switching in stacked $\mathrm{ZrO}_{\mathrm{x}} / \mathrm{ZrO}_{2} / \mathrm{ZrO}_{\mathrm{x}}$ structure AIP Adv. 635103

[15] Wu X, Zhou P, Li J, Chen L Y, Lv H B, Lin Y Y and Tang T A 2007 Reproducible unipolar resistance switching in stoichiometric $\mathrm{ZrO}_{2}$ films Appl. Phys. Lett. 90183507

[16] Lin C-C, Chang Y-P, Lin H-B and Lin C-H 2012 Effect of non-lattice oxygen on $\mathrm{ZrO}_{2}$-based resistive switching memory Nanoscale Res. Lett. 7187

[17] Liu Q, Guan W, Long S, Jia R, Liu M and Chen J 2008 Resistive switching memory effect of $\mathrm{ZrO}_{2}$ films with $\mathrm{Zr}+$ implanted Appl. Phys. Lett. 92012117

[18] Bousoulas P, Asenov P, Karageorgiou I, Sakellaropoulos D, Stathopoulos S and Tsoukalas D 2016 Engineering amorphous-crystalline interfaces in $\mathrm{TiO}_{2-x} / \mathrm{TiO}_{2-y^{-}}$ based bilayer structures for enhanced resistive switching and synaptic properties $J$. Appl. Phys. 120154501

[19] Tang Z, Fang L, Xu N and Liu R 2015 Forming compliance dominated memristive switching through interfacial reaction in $\mathrm{Ti} / \mathrm{TiO}_{2} / \mathrm{Au}$ structure J. Appl. Phys. 118 185309

[20] Lee M-J, Lee C B, Lee D, Lee S R, Chang M, Hur J H, Kim Y-B, Kim C-J, Seo D H, Seo S, Chung U-I, Yoo I-K and Kim K 2011 A fast, high-endurance and scalable nonvolatile memory device made from asymmetric $\mathrm{Ta}_{2} \mathrm{O}_{(5-\mathrm{x})} / \mathrm{TaO}_{(2-\mathrm{x})}$ bilayer structures . 
Nat. Mater. 10 625-30

[21] Huang C-Y, Huang C-Y, Tsai T-L, Lin C-A and Tseng T-Y 2014 Switching mechanism of double forming process phenomenon in $\mathrm{ZrO}_{\mathrm{x}} / \mathrm{HfO}_{\mathrm{y}}$ bilayer resistive switching memory structure with large endurance Appl. Phys. Lett. 10462901

[22] Ye C, Deng T, Zhang J, Shen L, He P, Wei W and Wang H 2016 Enhanced resistive switching performance for bilayer $\mathrm{HfO}_{2} / \mathrm{TiO}_{2}$ resistive random access memory Semicond. Sci. Technol. 31105005

[23] Ielmini D 2016 Resistive switching memories based on metal oxides: mechanisms, reliability and scaling Semicond. Sci. Technol. 31063002

[24] Baek G H, Lee A R, Kim T Y, Im H S and Hong J P 2016 Oxide stoichiometrycontrolled $\mathrm{TaO}_{\mathrm{x}}$-based resistive switching behaviors Appl. Phys. Lett. 109143502

[25] Jeon H, Park J, Jang W, Kim H, Kang C, Song H, Kim H, Seo H and Jeon H 2014 Stabilized resistive switching behaviors of a Pt/TaO $/$ /TiN RRAM under different oxygen contents Phys. Status Solidi 211 2189-94

[26] Lampert M A. 1956 Simplified theory of space-charge-limited currents in an insulator with traps Phys. Rev. 103 1648-56

[27] Chiu F-C 2014 A Review on Conduction Mechanisms in Dielectric Films Adv. Mater. Sci. Eng. 2014578168

[28] Xu Z, Yu L, Xu X, Miao J and Jiang Y 2014 Effect of oxide/oxide interface on polarity dependent resistive switching behavior in $\mathrm{ZnO} / \mathrm{ZrO}_{2}$ heterostructures Appl. Phys. Lett. 104192903

[29] Ma W, Herbert F W, Senanayake S D and Yildiz B 2015 Non-equilibrium oxidation states of zirconium during early stages of metal oxidation Appl. Phys. Lett. 106 101603

[30] Bakradze G, Jeurgens L P H and Mittemeijer E J 2011 The different initial oxidation kinetics of $\operatorname{Zr}(0001)$ and $\operatorname{Zr}(1010)$ surfaces J. Appl. Phys. 110024904

[31] Bespalov I, Datler M, Buhr S, Drachsel W, Rupprechter G and Suchorski Y 2015 Initial stages of oxide formation on the $\mathrm{Zr}$ surface at low oxygen pressure: An in situ FIM and XPS study Ultramicroscopy 159 147-51

[32] Li H, Choi J I J, Mayr-Schmölzer W, Weilach C, Rameshan C, Mittendorfer F, Redinger J, Schmid M and Rupprechter G 2015 Growth of an ultrathin zirconia film on $\mathrm{Pt}_{3} \mathrm{Zr}$ examined by high-resolution X-ray photoelectron spectroscopy, temperatureprogrammed desorption, scanning tunneling microscopy, and density functional theory J. Phys. Chem. C $1192462-70$

[33] Ismail M, Talib I, Huang C-Y, Hung C-J, Tsai T-L, Jieng J-H, Chand U, Lin C-A, Ahmed E, Rana A M, Nadeem M Y and Tseng T-Y 2014 Resistive switching characteristics of $\mathrm{Pt} / \mathrm{CeO}_{\mathrm{x}} / \mathrm{TiN}$ memory device Jpn. J. Appl. Phys $\mathbf{5 3} 060303$

[34] Kim J, Lee K, Kim Y, Na H, Ko D-H, Sohn H and Lee S 2013 Effect of thermal annealing on resistance switching characteristics of $\mathrm{Pt} / \mathrm{ZrO} 2 / \mathrm{TiN}$ stacks Mater. Chem. Phys. 142 608-613

[35] Morgan K A, Huang R, Pearce S, Zhong L, Jiang L and de Groot C H 2014 Effect of Stoichiometry of TiN Electrode on the Switching Behavior of TiN/HfO $/ \mathrm{TiN}$ Structures for Resistive RAM MRS Proc. 1631 mrsf13-1631-p03-01 